\title{
Photometric analysis of the optical counterpart of the black hole HMXB M 33 X-7 (Research Note)
}

\author{
A. Shporer ${ }^{1, \star}$, J. Hartman ${ }^{2}$, T. Mazeh ${ }^{1}$, and W. Pietsch ${ }^{3}$ \\ 1 Wise Observatory, Tel Aviv University, 69978 Tel Aviv, Israel \\ e-mail: shporer@wise.tau.ac.il \\ 2 Harvard-Smithsonian Center for Astrophysics, 60 Garden St., Cambridge, MA 02138, USA \\ 3 Max-Planck-Institut für extraterrestrische Physik, Giessenbachstrasse, 85741 Garching, Germany \\ Received 6 September 2006 / Accepted 26 November 2006
}

\section{ABSTRACT}

\begin{abstract}
Aims. Study the high-mass X-ray binary X-7 in M 33 using broad-band optical data.
Methods. We used recently published CFHT $r^{\prime}$ and $i^{\prime}$ data for variable stars in M 33 to extract the light curve of the optical counterpart of X-7. We combined these data with DIRECT $B$ and $V$ measurements in order to search for an independent optical modulation with the X-ray periodicity. The periodic modulation is modelled with the ellipsoidal effect. We used $U B V R r^{\prime} i^{\prime}$ magnitudes of the system to constrain the temperature and radius of the optical component.

Results. The optical data revealed a periodicity of $3.4530 \pm 0.0014$ days, which is consistent with the known X-ray period. Double modulation, which we attributed to ellipsoidal modulation, is clearly seen in four different optical bands. The absolute magnitude in six optical bands is most consistent with a stellar counterpart with $33000<T_{\text {eff }}<47000 \mathrm{~K}$ and $15<R<20 R_{\odot}$. We modelled the optical periodic modulation and derived the masses of the two components as a function of the orbital inclination and the radius of the stellar component. The resulting mass range for the compact object is $1.3<M<23 M_{\odot}$.

Conclusions. The system is probably a black hole HMXB, similar to Cyg X-1, LMC X-1 and LMC X-3.
\end{abstract}

Key words. stars: binaries: eclipsing - X-rays: individuals: M 33 X-7

\section{Introduction}

M 33 X-7 (hereafter X-7) is a well known bright variable X-ray source in the nearby M33 galaxy (Long et al. 1981). Its variability was identified as periodic by Peres et al. (1989), who derived a period of 1.7857 days and were the first to suggest its XRB nature. Using data from Einstein, ROSAT and ASCA, Larson \& Schulman (1997) have suggested that the actual period is 3.4531 days. This was confirmed by Dubus et al. (1999), who reported a period of $3.4535 \pm 0.0005$ days.

Pietsch et al. (2004) have analyzed X-ray data from XMM-Newton and Chandra and derived an improved period of $3.45376 \pm 0.00021$ days. They also used optical $B$ and $V$ data to suggest the optical companion is of spectral type between B0 I and $\mathrm{O} 7 \mathrm{I}$, with masses of $25-35 M_{\odot}$, making the compact binary component a black hole. If this is true, X-7 is the first eclipsing black hole high mass X-ray binary (HMXB).

In the course of the Chandra ACIS-I survey of M33 (ChASeM 33, Sasaki 2005), the X-ray eclipse ingress and egress of X-7 were observed for the first time (Sasaki et al. 2005). Pietsch et al. (2006) combined Einstein, ROSAT and XMMNewton data, and derived an improved period, of $3.453014 \pm$ 0.000020 days and a possible period decay rate of $\dot{P} / P=$ $-4 \times 10^{-6} \mathrm{yr}^{-1}$. Pietsch et al. (2006) have also used HST WFPC2

\footnotetext{
* Based on observations obtained with MegaPrime/MegaCam, a joint project of CFHT and CEA/DAPNIA, at the Canada-France-Hawaii Telescope (CFHT) which is operated by the National Research Council (NRC) of Canada, the Institute National des Sciences de l'Univers of the Centre National de la Recherche Scientifique of France, and the University of Hawaii.
}

images to resolve the OB association HS 13 (Humphreys \& Sandage 1980) in which X-7 resides, and have identified the optical counterpart to be an O6 III star whose mass is at least $20 M_{\odot}$. Based on the optical counterpart identification, possible orbital period decay rate, lack of pulsations, X-ray spectrum and fits to the optical light curves of Pietsch et al. (2004), Pietsch et al. (2006) suggested that X-7 is a black hole HMXB.

In the previous studies the periodicity of X-7 was derived from X-ray data, and no independent analysis of optical data was performed. Optical light curves of X-7 were presented in $B$ and $V$ filters by Pietsch et al. (2004), who folded the optical measurements on the X-ray period. This paper presents an analysis of optical light curves of the X-7 counterpart obtained by Hartman et al. (2006) in a search for variable stars in M33, performed at the Canada-France-Hawaii-Telescope (CFHT) with the Sloan $r^{\prime}$ and $i^{\prime}$ filters. Hartman et al. (2006) measurements are combined here with the $B$ and $V$ observations of Pietsch et al. (2004) to search independently for a periodic modulation. The combined data, as presented in Sect. 2 do show clear periodicity with the X-ray period, as presented in Sect. 3. Based on broad-band $U B V R r^{\prime} i^{\prime}$ data from Pietsch et al. (2006), Massey et al. (2006) and Hartman et al. (2006) we apply a photometric modelling to derive the stellar temperature and radius of the optical component in Sect. 4.1, and periodic ellipsoidal model in Sect. 4.2. We briefly discuss our results in Sect. 5 .

\section{The optical data}

The present analysis is based on the optical measurements of X-7 in four bands, obtained from two sets of observations carried out 


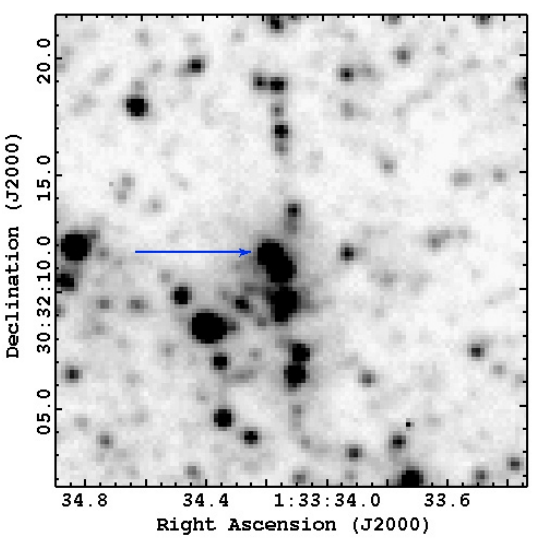

Fig. 1. X-7 finding chart, in $i^{\prime}$, from the CFHT data. Position of the variable object is indicated by an arrow. Image FOV is $20 \times 20$ arcsec. North is up and East is to the left.

$\sim 4$ years apart. The $B$ and $V$ light curves of Pietsch et al. (2004, Table 4) were obtained from a special photometric analysis of DIRECT $^{1}$ (Mochejska et al. 2001) images, taken at the Kitt Peak National Observatory 2.1-m telescope in 1999. The $V$ light curve includes 70 measurements and the $B$ light curve only 30 points. Sloan $r^{\prime}$ and $i^{\prime}$ light curves were taken from the publicly available data of the M 33 CFHT variability survey ${ }^{2}$ (Hartman et al. 2006, object 243718). Their data were obtained at the CFHT 3.6-m telescope atop Mauna Kea on two consecutive observing seasons in 2003 and 2004. An $i^{\prime}$ finding chart of X-7 is given in Fig. 1. A comparison with the finding chart of Pietsch et al. (2006, their Fig. 5) shows this object is at the position of X-7 optical counterpart identified by Pietsch et al. (2006). The identification of the same period in the optical as in the X-rays (presented hereafter) confirms the identification. The $i^{\prime}$ light curve contains 32 measurements, where we ignored two measurements with uncertainty larger than 0.04 mag. The $r^{\prime}$ light curve consists of 31 measurements, where we ignored three obvious outliers.

\section{Period analysis}

To search for periodicity we have applied a multi-band doubleharmonic fitting (Shporer \& Mazeh 2006) to the four available light curves together, which included 158 individual measurements. For each trial period we fitted the entire data set with a double-harmonic function, with independent zero points for each of the four light curves. The periodogram value for each trial period was taken as the power (sum of squared harmonic coefficients) divided by the $\chi^{2}$ goodness-of-fit parameter of the fitted light curve.

Figure 2 presents the resulting periodogram. The strongest peak corresponds to a period of $P_{\mathrm{opt}}=3.4530 \pm 0.0014$ days. The second strongest peak is exactly at the first harmonic of $P_{\text {opt }}$. The third strongest peak is at a frequency corresponding to the lunar cycle. The optical period agrees well with the known X-rays period, $P_{\mathrm{X}}=3.453014 \pm 0.000020$ days, of Pietsch et al. (2006).

Figure 3 presents the four light curves folded on the detected period, with phase zero taken at HJD $=2453639.119$ - the mideclipse time of Pietsch et al. (2006). The figure shows the $B$ and $V$ data of Pietsch et al. (2004) for completeness, including seven $V$ measurements that were not plotted in Fig. 4 of Pietsch et al. (2004), since that figure brings only $V$ measurements that

\footnotetext{
1 http://cfa-www.harvard.edu/ kstanek/DIRECT/

${ }^{2}$ http://www.astro.livjm.ac.uk/ dfb/M 33/
}

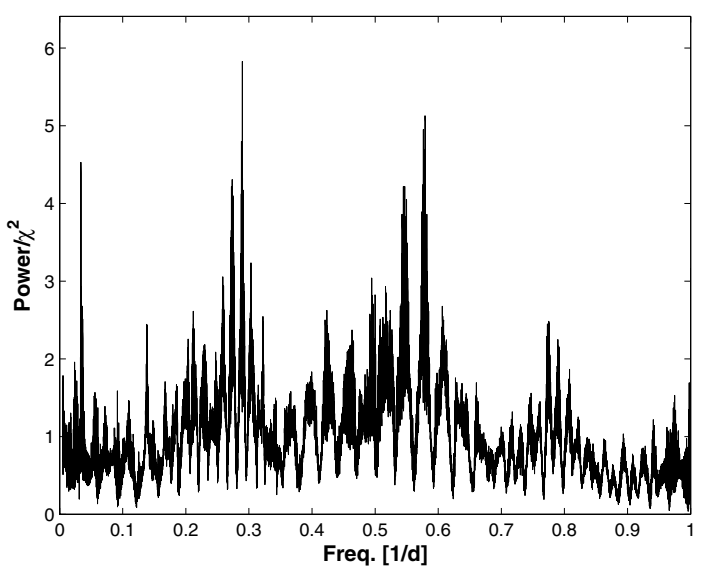

Fig. 2. Multi-band double-harmonic periodogram.
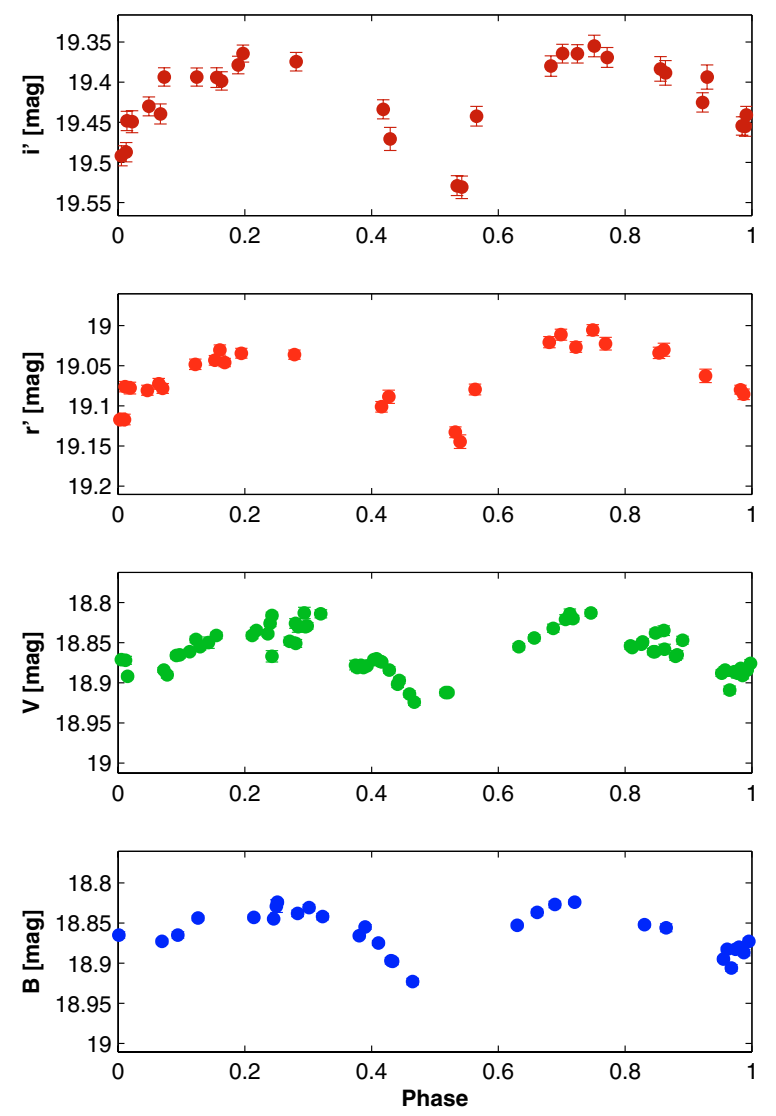

Fig. 3. Folded light curves, in magnitude, in (top to bottom) $i^{\prime}, r^{\prime}, V$ and $B$. All light curves are folded on the period identified here, of 3.4530 days, and phase zero was taken to be at HJD $=2453639.119$, which is time of mid-eclipse in the X-rays (Pietsch et al. 2006).

were obtained close to $B$ measurements. Figure 3 clearly shows ellipsoidal modulation in all four bands. For each band independently, we fitted the data with two harmonics and derived the total amplitude of the modulation and the phase of minimum. Results are listed in Table 1. For the $i^{\prime}$ and $r^{\prime}$ bands we ignored the four faintest points in each of these bands. The amplitudes derived when we included these points in the analysis are given in parenthesis.

It appears as if the amplitude of the modulation decreases with decreasing wavelength, from $i^{\prime}$ to $B$, even when ignoring the four faintest points in $i^{\prime}$ and $r^{\prime}$. The origin of this varying modulation is not clear. It could result from some real wavelength 
Table 1. Harmonic fits amplitude and minimum phase near 0.0.

\begin{tabular}{cll}
\hline \hline Filter & $\begin{array}{c}\text { Amp. } \\
{[\mathrm{mmag}]}\end{array}$ & Min. Phase \\
\hline$i^{\prime}$ & $47 \pm 3(63 \pm 4)$ & $0.962 \pm 0.006(0.949 \pm 0.006)$ \\
$r^{\prime}$ & $39 \pm 2(48 \pm 2)$ & $0.960 \pm 0.003(0.953 \pm 0.003)$ \\
$V$ & $34 \pm 1$ & $0.970 \pm 0.001$ \\
$B$ & $30 \pm 1$ & $0.979 \pm 0.001$ \\
\hline
\end{tabular}

dependence of the system's modulation. On the other hand, the different modulation could come either from some secular changes during the $\sim 4$ years between the KPNO $B$ and $V$ and CFHT $i^{\prime}$ and $r^{\prime}$ observations, or from some wavelengthdependent blending of light from a near-by star.

\section{Modelling the system}

In our modelling of the X-7 binary system, we preferred to find first a stellar model that fits the broad-band photometry of the system, assuming the optical brightness is coming from the optical star alone. From the stellar radius, the observed X-ray eclipse width and the periodic ellipsoidal modulation we can derive the two masses as a function of the orbital inclination.

\subsection{Optical star modelling}

We have used the following broad-band magnitudes: $U=18.1$, $B=18.8, V=18.9$ (Pietsch et al. 2006, based on HST data), $R=$ 19.0 (Massey et al. 2006, $\mathrm{LGGS}^{3}$ ), $r^{\prime}=19.1$ and $i^{\prime}=19.42 \mathrm{mag}$ (Hartman et al. 2006), assuming an uncertainty of 0.1 mag on all values. We converted the observed measurements to absolute magnitudes by using an M33 distance modulus of 24.62 mag (Freedman et al. 2001), $A_{\mathrm{V}}=0.53 \pm 0.06 \mathrm{mag}$ from Pietsch et al. (2006) and $A_{\lambda} / A_{\mathrm{V}}$ values from Schlegel et al. (1998).

We compared these magnitudes to the ones determined from the integrated magnitudes from Girardi et al. (2002, 2004), where we assume $M_{\text {bol, } \odot}=4.77$ (Girardi et al. 2002) and fit for the radius for each value of $\log (g)$ and $T_{\text {eff }}$. We did this for both $[\mathrm{Fe} / \mathrm{H}]=0$ and $[\mathrm{Fe} / \mathrm{H}]=-0.5$ tables, since assuming $\mathrm{X}-7$ is at a galactocentric distance of $2 \mathrm{kpc}$ results in $[\mathrm{Fe} / \mathrm{H}]=-0.22$, based on the $[\mathrm{O} / \mathrm{H}]$ gradient of Garnett et al. (1997) and the $[\mathrm{O} / \mathrm{H}]$ to $[\mathrm{Fe} / \mathrm{H}]$ conversion of Maciel et al. (2003).

The data allow a large range of stellar models. One such model, for $[\mathrm{Fe} / \mathrm{H}]=0$, with $\chi^{2}=1.62$, has $T_{\text {eff }}=26000 \mathrm{~K}$, $\log g=5.0$ and $R=25 R_{\odot}$. For $[\mathrm{Fe} / \mathrm{H}]=-0.5$, with $\chi^{2}=1.55$, we get $T_{\text {eff }}=27000 \mathrm{~K}, \log g=5.0$ and $R=25 R_{\odot}$. The $95 \%$ uncertainty range, where $\chi^{2}$ increases by up to 8.0 (for three parameters), is: $18000<T_{\text {eff }}<47000 \mathrm{~K}, 15<R<35 R_{\odot}$. The value of $\log g$ is unconstrained. Figure 4 brings three representative models; the solid line presents the model with $T_{\text {eff }}=$ $27000 \mathrm{~K}, R=25 R_{\odot}$, the dotted line represent the model with $T_{\text {eff }}=18000 \mathrm{~K}, R=35 R_{\odot}$ and the dashed line shows the model with $T_{\text {eff }}=47000 \mathrm{~K}, R=15 R_{\odot}$. The figure shows that the absolute magnitudes of the X-7 optical counterpart can not constrain the stellar radius, and any value in the range of $15-35 R_{\odot}$ is possible. However, as we show in the next section, a stellar radius of $R>20 R_{\odot}$ is very unlikely, since the star will be too massive. Therefore, our most likely models are in the range of $15<R<20 R_{\odot}$ and $33000<T_{\text {eff }}<47000 \mathrm{~K}$.

3 The Local Group Galaxy Survey, http: //www . lowell . edu/users/massey/lgsurvey/index .html

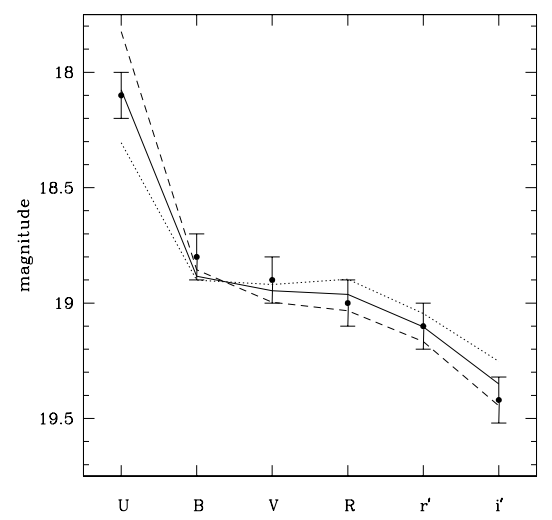

Fig. 4. Photometric broad-band modelling of the X-7 stellar component. Error bars present the assumed uncertainty of $0.1 \mathrm{mag}$ in all bands. Solid line presents a model with $T_{\text {eff }}=27000 \mathrm{~K}, \log g=5.0$ and $R=25 R_{\odot}$. The dashed and dotted lines present models at $95 \%$ confidence boundary of our modelling. The dotted line is a model with $T_{\text {eff }}=18000 \mathrm{~K}$, $R=35 R_{\odot}$, the dashed line is a model with $T_{\text {eff }}=47000 \mathrm{~K}, R=15 R_{\odot}$.

\subsection{Light-curve modelling}

We used two different codes, PHOEBE and ELC, to model the obtained periodic light curves and derive an estimate of the masses of both components. For both codes, the main effect is due to the tidally induced ellipsoidal shape of the optical component.

The first program is the "PHysics Of Eclipsing BinariEs" program of Prša \& Zwitter (2005), a front-end code for the Wilson-Devinney program (Wilson \& Devinney 1971; Wilson 1979, 1990). Following Pietsch et al. (2006), who used PHOEBE to model the $\mathrm{X}-7 \mathrm{~B}$ and $V$ light curves, we used the eclipse half angle value of $26.5^{\circ} \pm 1.1^{\circ}$ to find the ratio of the radius of the optical component to the semi-major axis as a function of inclination. We varied the inclination between $90^{\circ}$ and about $70^{\circ}$ and fit for the mass ratio and a luminosity scale to match each light curve, assuming a temperature of $27000 \mathrm{~K}$ and linear limbdarkening coefficients from Claret (2000, 2004), assuming a metallicity of $[\mathrm{Fe} / \mathrm{H}]=-0.2$.

We performed the fits ignoring the four faintest points in each of the $r^{\prime}$ and $i^{\prime}$ light curves which occur near phases 0 and 0.5 - no model was able to match these points. Including those points does not substantially change the mass-ratio, while it does slightly affect the luminosity scale.

There is not enough data to constrain the stellar radius or the inclination of the system; a good fit to the light curves could be found for large ranges of these two parameters. One such model is plotted in Fig. 5. The PHOEBE light curve is drawn with a dotted line and the other model with a dashed line. For both, values of $R=20 R_{\odot}, T_{\text {eff }}=33000 \mathrm{~K}$ and $i=80^{\circ}$ were taken, although changing these values results in essentially no difference for the model light curve.

The range of allowed inclinations and stellar radii did not enable us to constrain the masses of the two components and they could only be derived as a function of the assumed orbital inclination and optical radius. We found that for the PHOEBE code the mass ratio $\left(M_{x} / M_{\mathrm{opt}}\right)$ varies from 0.29 at $90^{\circ}$ to 0.15 at $70^{\circ}$. Below $70^{\circ}$ the optical component would exceed its Roche lobe. Assuming $R=20 R_{\odot}$, the mass of the optical component would vary between $79 M_{\odot}$ and $49 M_{\odot}$, while that of the compact object would vary between $23 M_{\odot}$ and $7.5 M_{\odot}$. Because the fits are fairly insensitive to the temperature of the optical component, the resulting masses scale as the radius of the star to 

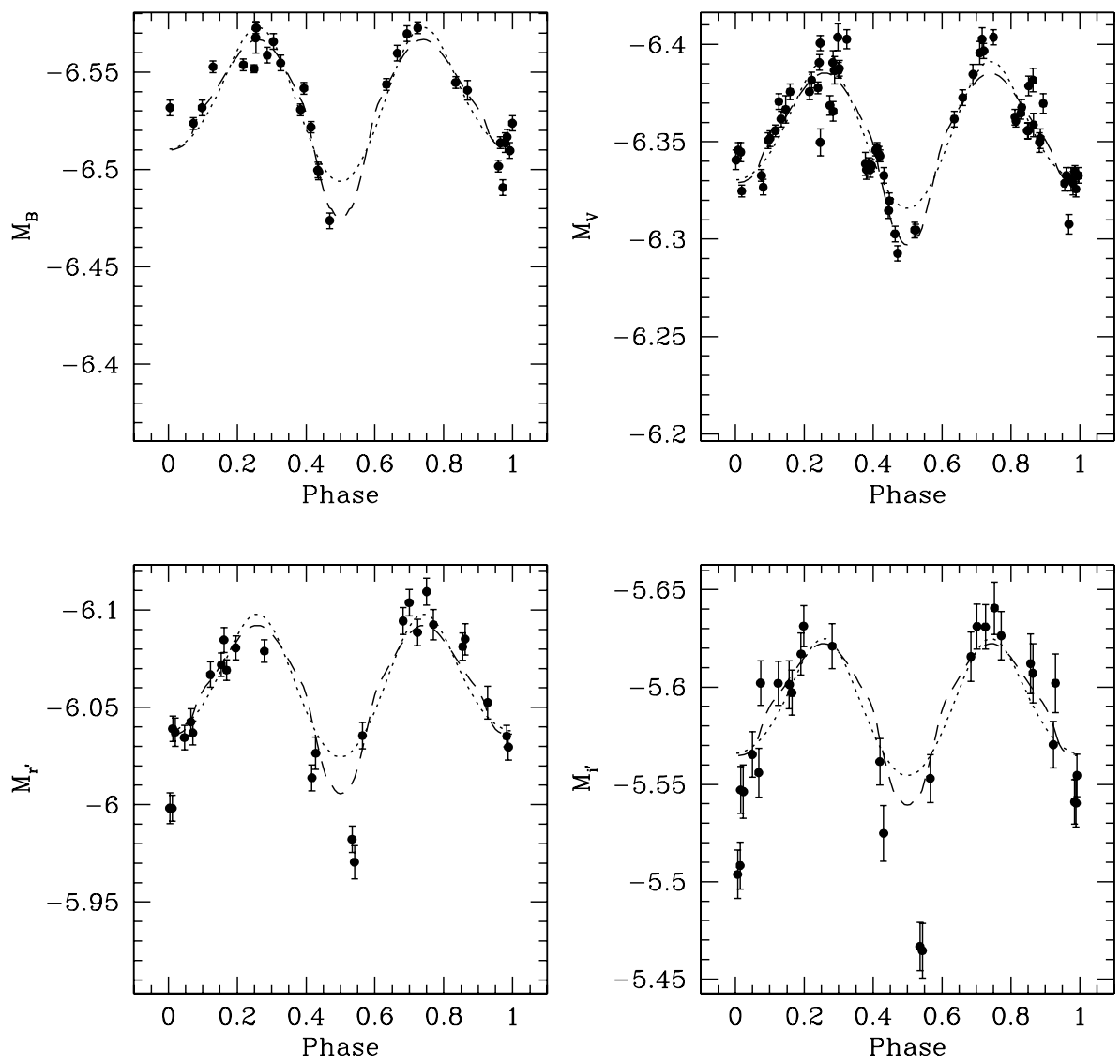

Fig. 5. $B V r^{\prime} i^{\prime}$ light curves phased at $P=$ 3.453014 day and $T_{0}=2453639.119$ from Pietsch et al. (2006), with model light curves for $R=20 R_{\odot}, T_{\text {eff }}=33000 \mathrm{~K}$ and $i=80^{\circ}$, overplotted. The dotted line is the PHOEBE light curve, with no accretion disk or X-ray heating. The dashed line is the ELC model which includes an accretion disk and X-ray heating.

the third power. For $R=15 R_{\odot}$, the mass of the optical component varies between $33 M_{\odot}$ and $21 M_{\odot}$, while the compact object mass varies between $10 M_{\odot}$ and $3 M_{\odot}$. We note, however, that assuming a stellar radius larger than $20 R_{\odot}$ results in an unrealistic large stellar mass. We therefore limit the discussion to stellar radii smaller than $20 R_{\odot}$.

The second program used was the ELC code of Orosz \& Hauschildt (2000). This program has been specifically tailored to model optical light curves of X-ray binary systems, including effects such as the presence of an accretion disk, X-ray heating of the optical component and the ability to explicitly take one of the components to be invisible. To model the X-ray heating we assumed an average X-ray luminosity of $10^{37.5} \mathrm{erg} \mathrm{s}^{-1}$, and to model the disk we assumed an inner disk temperature of $10^{7} \mathrm{~K}$ (Pietsch et al. 2006). We assumed a disk opening angle of $2^{\circ}$, and a power-law exponent for the temperature profile of the disk of -0.75 . We used the black-body mode of the model rather than using the detailed model atmosphere since the model atmosphere would not necessarily be a good match to the Sloan $r^{\prime}$ or $i^{\prime}$ filters. We proceeded as above, stepping through a range of inclination angles for two values of the optical radius and fitting for the mass ratio. This time we also fit for the inner and outer disk radii. One possible model, for $R=20 R_{\odot}, T_{\mathrm{eff}}=33000 \mathrm{~K}$ and $i=80^{\circ}$, is plotted in Fig. 5.

The resulting mass ratio varies from 0.23 at $90^{\circ}$ inclination to 0.07 at $65^{\circ}$ inclination. For a radius of $20 R_{\odot}$ this corresponds to a mass range of 83 to $42 M_{\odot}$ for the optical component and a range of 19 to $3 M_{\odot}$ for the compact object. Below $65^{\circ}$ the ELC model star would exceed its Roche lobe. In units of the inner Lagrange radius of the compact object, the inner disk radius varies from $1.6 \times 10^{-5}$ to $5.4 \times 10^{-5}$ while the outer disk radius varies from 0.61 to 0.81 .
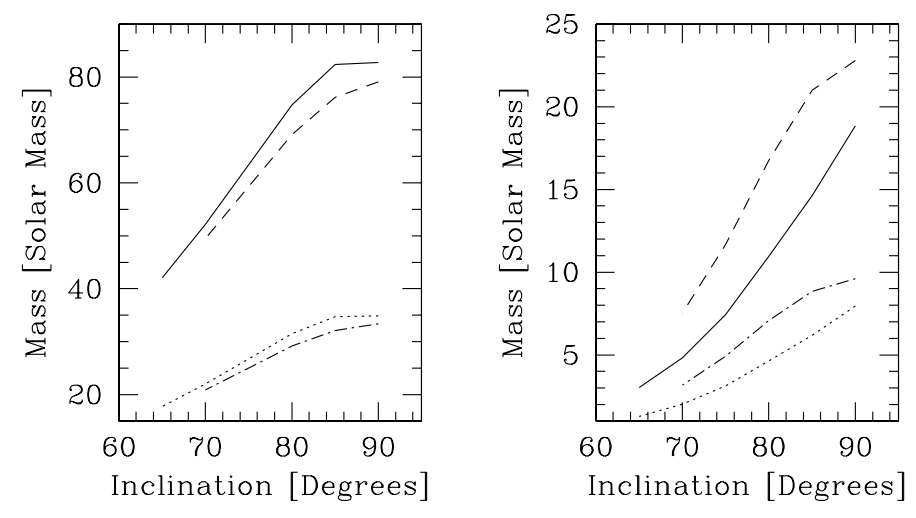

Fig. 6. Plots showing the mass vs. inclination for two values of the stellar radius. The left plot is for the optical component, the right plot is for the compact object. The solid and the dashed lines show the results for $R=20 R_{\odot}$, using the ELC and the PHOEBE codes, respectively. The dotted and the dot-dashed lines show the results for $R=15 R_{\odot}$, using the ELC and the PHOEBE codes, respectively.

The masses of both components as a function of inclination, derived by both codes for two values of the optical radius, are plotted in Fig. 6.

\section{Discussion}

We have conducted a broad-band optical analysis of the X-7 optical counterpart, including period analysis, photometric modelling and light curve modelling. We applied a multi-band double-harmonic period analysis of X-7 optical light curves in four bands and identified a period of $P_{\mathrm{opt}}=3.4530 \pm 0.0014$ days. This period, derived from an independent optical analysis, is in 
good agreement with the known X-rays period (Pietsch et al. 2006).

Using photometric absolute magnitudes in six bands, we find a range of models for the optical counterpart, with $15<R<$ $35 R_{\odot}$ and $18000<T_{\text {eff }}<47000 \mathrm{~K}$. However, stellar radius larger than $20 R_{\odot}$ results in an unrealistic large stellar mass. We therefore suggest that the stellar radius is smaller than $20 R_{\odot}$. The possible temperature range is reduced accordingly to $33000<$ $T_{\text {eff }}<47000$. The classification of the optical star as O6 III by Pietsch et al. (2006) is well within our uncertainties.

We have modelled the optical light curve using two programs. While the ELC model, incorporating both a disk and X-ray heating matches better to the data than the PHOEBE model (see Fig. 5), neither model yielded a good fit to the light curves. In particular, the minima in $r^{\prime}$ and $i^{\prime}$ appear to be underestimated by all models that we have tried. At this point it is unclear if this is due to random or systematic errors in the observations or to inadequacies in the physical models.

The simplistic models can nevertheless yield a rough estimate of the masses of the two components as a function of the optical radius and the orbital inclination. For the smallest likely optical radius $\left(15 R_{\odot}\right)$ we get $3 M_{\odot}$ and $1.3 M_{\odot}$ from the PHOEBE and ELC codes respectively. Therefore, the present analysis can not exclude completely a neutron star as the compact object, although this option is very unlikely.

On the other hand, the similarity between M33 X-7 and the three known black-hole HMXB - Cyg X-1, LMC X-1 and LMC X-3 (e.g., Cowley 1992; McClintock \& Remillard 2003), is striking. All four systems have orbital periods of a few days, their optical counterpart is an early-type star and the compact object has a mass of 6-10 $M_{\odot}$. Radial velocity measurements of M 33 X-7 will enable us to better constrain the masses of the two components. Large telescopes and presently efficient spectrographs render such measurements feasible. Together with the $\mathrm{X}$-ray eclipse and the optical light curves, we should be able to understand this system better than any other black-hole HMXB.

Finally, if this system would be proven to be a black-hole HMXB, it is interesting to note that three out of the four such known systems were found in external galaxies. This might indicate that we are not very efficient in detecting such systems in our own galaxy. Maybe the dust in the Galactic plane hides from our telescopes many more Galactic black holes.

Acknowledgements. We are grateful to J. McClintock for helpful discussions and to J. Orosz for sharing his ELC program with us. We thank the anonymous referee for his helpful comments. This work was partially funded by the German-Israeli Foundation for Scientific Research and Development and by the Israeli Science Foundation. This research has made use of NASA's Astrophysics Data System Abstract Service and of the SIMBAD database, operated at CDS, Strasbourg, France.

\section{References}

Claret, A. 2000, A\&A, 363, 1081

Claret, A. 2004, A\&A, 428, 1001

Cowley, A. P. 1992, ARA\&A, 30, 287

Dubus, G., Charles, P. A., Long, K. S., Hakala, P. J., \& Kuulkers, E. 1999, MNRAS, 302, 731

Freedman, W. L., Madore, B. F., Gibson, B. K., et al. 2001, ApJ, 553, 47

Garnett, D. R., Shields, G. A., Skillman, E. D., Sagan, S. P., \& Dufour, R. J. 1997, ApJ, 489, 63

Girardi, L., Bertelli, G., Bressan, A., et al. 2002, A\&A, 391, 195

Girardi, L., Grebel, E. K., Odenkirchen, M., \& Chiosi, C. 2004, A\&A, 422, 205

Hartman, J. D., Bersier, D., Stanek, K. Z., et al. 2006, MNRAS, 371, 1405

Humphreys, R. M., \& Sandage, A. 1980, ApJS, 44, 319

Larson, D. T., \& Schulman, E. 1997, AJ, 113, 618

Long, K. S., Dodorico, S., Charles, P. A., \& Dopita, M. A. 1981, ApJ, 246, L61

Maciel, W. J., Costa, R. D. D., \& Uchida, M. M. M. 2003, A\&A, 397, 667

Massey, P., Olsen, K. A. G., Hodge, P. W., et al. 2006, AJ, 131, 2478

McClintock, J. E., \& Remillard, R. A. 2003 [arXiv:astro-ph/0306213]

Mochejska, B. J., Kaluzny, J., Stanek, K. Z., Sasselov, D. D., \& Szentgyorgyi, A. H. 2001, AJ, 122, 2477

Orosz, J. A., \& Hauschildt, P. H. 2000, A\&A, 364, 265

Peres, G., Reale, F., Collura, A., \& Fabbiano, G. 1989, ApJ, 336, 140

Pietsch, W., Mochejska, B. J., Misanovic, Z., et al. 2004, A\&A, 413, 879

Pietsch, W., Haberl, F., Sasaki, M., et al. 2006, ApJ, 646, 420

Prša, A., \& Zwitter, T. 2005, ApJ, 628, 426

Sasaki, M. 2005, in Chandra Proposal

Sasaki, M., Pietsch, W., Haberl, F., et al. 2005, The Astronomer's Telegram, 633

Schlegel, D. J., Finkbeiner, D. P., \& Davis, M. 1998, ApJ, 500, 525

Shporer, A., \& Mazeh, T. 2006, MNRAS, 370, 1429

Wilson, R. E. 1979, ApJ, 234, 1054

Wilson, R. E. 1990, ApJ, 356, 613

Wilson, R. E., \& Devinney, E. J. 1971, ApJ, 166, 605 